\title{
A Brunswikian evolutionary developmental theory of preparedness and plasticity
}

\author{
Aurelio José Figueredo $^{1}$, Kenneth R. Hammond ${ }^{2}$, and Erin C. McKiernan ${ }^{3}$ \\ ${ }^{1}$ Ethology and Evolutionary Psychology, University of Arizona, Tucson, \\ AZ, USA \\ ${ }^{2}$ Center for Research on Judgment and Policy, University of Colorado, \\ Boulder, CO, USA \\ ${ }^{3}$ Arizona Research Laboratories, Division of Neurobiology, University of \\ Arizona, Tucson, AZ, USA
}

\begin{abstract}
The domain-independent and domain-dependent approach to the evolution of cognition have been taken by separate groups of researchers who have focused exclusively on either the formal properties or the distinct cognitive demands of tasks. We express the view that synthesizing the two approaches could lead to a more complete understanding, and propose such a comprehensive model of cognitive evolution and development. First, we discuss how Egon Brunswik demonstrated the importance of the relationship between the organism and the environment, and how his research and that of others has led to the domain-independent and domain-dependent views. Second, we use Brunswikian concepts to propose a twoparameter evolutionary model of cognitive development that specifies how particular behaviors come to be characterized by independent levels of biological preparedness and developmental plasticity. Our theory incorporates both a domainindependent organizing principle and the importance of domain-dependent processes. Third, we briefly discuss one unique prediction arising from the Brunswikian Evolutionary Developmental theory and describe preliminary supporting evidence.
\end{abstract}

Keywords: Domain-independent; Domain-dependent; Cognition; Biological preparedness; Developmental plasticity; Extinction; Habituation; Associative learning; Nonassociative learning 


\section{Introduction}

Any comprehensive treatment of the evolution of cognition should take into account the formal properties of tasks and distinguish the different cognitive demands made by each task in different environmental contexts. The domain-independent approach and the domain-dependent approach taken, at least to date, by separate groups of researchers, have focused exclusively on only one of these major facets of cognition. The domain-independent approach emphasizes the formal operating characteristics that are shared by many cognitive processes, whereas the domaindependent approach focuses on the unique characteristics of different cognitive processes. It is our view that synthesizing the two approaches could lead to a more complete understanding of cognition. Such a comprehensive model of cognitive evolution and development is proposed herein.

The argument is divided into three parts. First, we discuss how Egon Brunswik anticipated basic assumptions of evolutionary psychology by demonstrating the importance of the relationship between the organism and the environment. His research and that of others has led to the domain-independent and domain-dependent views. Second, we utilize concepts introduced by Brunswik to propose a twoparameter evolutionary model of cognitive development that specifies how particular behaviors come to be characterized by a certain level of biological preparedness and a certain independent level of developmental plasticity. The proposed Brunswikian Evolutionary Developmental theory utilizes a domain-independent organizing principle (the Lens Model) and takes into account the influence of domain-dependent utilization by the organism of ecologically valid stimuli and responses (cues and means). Third, we discuss one prediction arising from the Brunswikian Evolutionary Developmental theory: the hypothesis that two observable behavioral mechanisms, extinction and habituation, commonly thought of as distinct associative and nonassociative learning processes, may represent fundamentally the same learning process. Our view is that the merger of the domainindependent and domain-dependent views accomplished here reveals that the cognitive task the brain must solve in both extinction and habituation is essentially the same. Preliminary evidence in support of this prediction is discussed briefly and in detail by McKiernan and Figueredo (in preparation). Finally, we express the hope that researchers in many areas will benefit from thinking about domainindependence and domain-dependence as complementary rather than mutually exclusive developmental mechanisms.

\section{Brunswikian psychology and domain-independence}

Brunswik's Probabilistic Functionalism, as it appears today in the work of many authors, clearly entails a domain-independent approach to cognition, specifically, to judgment under uncertainty. By including the term "probabilistic" he became the first psychologist to acknowledge the role of uncertainty in the environment

Author version. Final version published in Intelligence, v.34: 211-227 available at www.sciencedirect.com/science/article/pii/S016028960500022X.

DOI: $10.1016 /$ j.intell.2005.03.006 
(see Brunswik, 1952, 1955a, 1956, 1957), as well as the first to describe in detail - and carry out an experiment (Brunswik, 1938; see Doherty, 2001) - to show the consequences of that idea for a theory of learning.

Probabilistic Functionalism, as developed by Brunswik (1952, 1955b, 1956), clearly distinguishes between (a) the proximal stimulation received by an organism and the distal state of the environment that ultimately produced it, and (b) the proximal reaction or response effected by an organism and the distal achievement that it, in turn, ultimately produces. For that reason, proximal stimuli are often referred to in this model as cues to external states and proximal responses as means to external ends. This distinction recognizes that there is often an array of multiple proximal cues available that indicate any given distal event, as well as an array of multiple proximal means available that might converge upon any given distal achievement. These arrays of cues and means are represented graphically in Fig. 1 by diverging and converging sets of arrows. The lens itself is used to represent the interface between the organism and the environment. Cues, or "stimuli", are represented by the array of arrowheads directed from the environment to the organism; means, or "responses", are represented by the array of arrowheads directed from the organism to the environment.

Both cues and means may differ appreciably in either their veridicality as indicators or their instrumentality as effectors. The ecological validity of any given cue (e.g. waist-hip ratio) is the association between a proximal stimulus received (e.g. the observed waist-hip ratio of a particular woman) and the distal event (e.g. that woman's fertility) presumably indicated in the environment, regardless of the behavioral utilization of that cue by the organism; that of any given means (e.g. courting strategy) is the association between a proximal response effected (e.g. use of that courting strategy) and the distal achievement (e.g. successful copulation) presumably produced in the environment, regardless of utilization of that means by the organism. The functional utilization of any given cue is the association between a proximal stimulus received and behavioral utilization by the organism of that cue as an indicator of the specified distal event, regardless of the objective state of the environment; that of any given means is the association between a proximal response effected and its use by the organism as an effector of the specified distal achievement, regardless of the causal effectiveness of that means on the environment.

Thus, a full distal stimulus-response (S-R) association is mediated by four linking coefficients: (1) the ecological validity with which a given cue indicates a state of the environment, (2) the functional utilization with which the given proximal stimulus is used by the organism, (3) the functional utilization with which the given proximal response is used by the organism, and (4) the ecological validity with which that means produces the specified effect on the environment. These four linking pathways may be traced in Fig. 1 by following the arrows from the environment, through the lens, and into the organism, then back from the organism, through the lens, and out to the environment. Alternative representations, such as the double lens model of Petrinovich (1979), make this complex mediation more

Author version. Final version published in Intelligence, v.34: 211-227 available at www.sciencedirect.com/science/article/pii/S016028960500022X. DOI: $10.1016 /$ j.intell.2005.03.006 
explicit.

\subsection{The lens model as a domain-independent cognitive process}

The domain-independent theory of cognitive processing represented by the Lens Model goes back as far as Tolman and Brunswik's landmark paper that expanded psychological theory to include specific characteristics of the environment. Brunswik and Tolman began their work by first describing the properties of the environmental circumstances with which the organism must cope, and then deduced the properties of the "psychological mechanisms" (meaning proximate processes of mediation) that must necessarily be possessed by surviving organisms. They initially postulated a "textured" environment that mediates information in uncertain, interdependent terms, and then formulated a theory of cognitive processes that could cope with probabilistic, interdependent information. The Lens Model is domainindependent because it is designed to deal with any information of this nature and not just with the specific characteristics of any particular cognitive task.

Brunswik's postulation of a probabilistic, multiple-cue environment came from his pains- taking studies of visual perception, in particular, the phenomenon of size constancy carried out in Karl Buhler's laboratory in Vienna as far back as the early 1930s (see Brunswik, 1952, 1956). It was this work that turned up the various probabilistic, interdependent cues to size, and other distal variables in the natural environment (Tolman \& Brunswik, 1935). The term "Causal Texture of the Environment" indicated the entangled or intercorrelated relations among perceptual cues such as perspective, interception, vertical position and others and their less-than-perfect relation to distance or size. Because size constancy is critical to survival, they hypothesized that organisms would have been naturally selected to be able to make use of such imperfect data (i.e., would have evolved a "psychological mechanism" that would enable them to do so). This hypothesis led Brunswik to carry out the first rat experiment on probabilistic learning at Berkeley in 1939 (see Doherty, 2001) and others subsequently, to illustrate the point (Brunswik \& Herma, 1951; for comments see Bjorkman, 2001). By 1956 Brunswik had decided that the multiple regression model was a reasonable "psychological mechanism" for coping with such tasks (Brunswik, 1956, p. 110) because it is based on data from several variables, all of which have, (a) imperfect relations with the criterion in question, (b) may have imperfect redundant relations with one another, (c) have a functional relationship with the criterion which may take on various non-linear (as well as linear) forms, and (d) the equation provides an overall measure of predictability (the multiple $\mathrm{R}$ value). For this reason, the Lens Model is often also referred to interchangeably as the "Linear Model". This nomenclature does not imply that all statistical relations modeled must necessarily be rectilinear in the geometric sense, but refers to the statistical format of the general linear model, which can also handle curvilinear terms (Cohen \& Cohen, 1983).

Author version. Final version published in Intelligence, v.34: 211-227 available at www.sciencedirect.com/science/article/pii/S016028960500022X.

DOI: $10.1016 / j$.intell.2005.03.006 


\subsection{Fallible indicators and the linear model}

The fit between theory and quantitative expression was a natural one, and it is worth noting that Brunswik first developed the theory and then noted its parallel to the multiple regression equation, not the reverse (see Gigerenzer, 1991, for many examples of the reverse procedure). Brunswik's evolutionary psychology thus begins with an empirically based theory of the general characteristics of environmental tasks that challenge organismic perception in general and human judgment in particular. It argues that the natural environment offers a wide variety of interdependent, fallible, or probabilistic, indicators of events or environmental states that are not directly observable. It is these ubiquitous fallible indicators - not limited to those involved in the visual perception of size, color, and shape that intrigued Brunswik - that must be organized to form the inductive inferences, the judgments that an organism must make about distal variables in order to survive in a probabilistic environment.

There is an abundance of empirical laboratory research that illustrates the capacity of humans and numerous other species of nonhuman animals to make use of multiple fallible indicators widely varying in content for such inferences (see Holzworth, 2001). Albright and Malloy (2001), Funder (2001), Gillis and Bernieri (2001), and Mumpower (2001) make the case for the pervasive use of multiple fallible indicators and the linear model in social perception (an area Brunswik opened up in the 1940s). Buss (1989) illustrates this mechanism in mate selection. Moreover, there is abundant evidence of the use of multiple fallible indicators (e.g., wave direction) by migrating species that range from pigeons to turtles. Byrne (1995) describes the use of multiple fallible indicators by apes as a means of group communication about the location of food. Von Schantz et al. (1997) conducted painstaking field studies of female pheasants' use of multiple fallible indicators in their choice of mates and found that the linear model successfully accounted for the female pheasants' accurate choices about which male mating would result in the production of the largest number of viable eggs. This discovery provides extraordinary support for the linear model as a heritable genetic trait. There is no obvious means by which the pheasant could learn to accomplish this feat, and the increased number of viable eggs provides evidence of a heritable mechanism. Although no direct studies have been done on the inheritance of the use of multiple and fallible indicators within a species, its widespread appearance across species offers plausibility for the linear model as a general framework for cognition.

The functional reason why the linear model has been found to be so general over the wide variety of content of the fallible indicators, from those used in studies carried out in the psychological laboratory to those found by behavioral ecologists, is its robustness: a feature of the linear model made apparent to judgment and decision researchers by Dawes and Corrigan (1974). The ability of this form of aggregation to produce an approximately correct judgment even when it includes incorrect cue weights or function forms, or even the incorrect aggregation rule, is a truly startling result. Such robustness was well-known to statisticians, but the

Author version. Final version published in Intelligence, v.34: 211-227 available at www.sciencedirect.com/science/article/pii/S016028960500022X.

DOI: $10.1016 / j$.intell.2005.03.006 
implications of the robustness of the linear model as a cognitive model for a wide variety of species - pheasants, turtles and even mosquitoes (see Wilton, 1968) - has never been sufficiently emphasized by evolutionary psychologists or biologists.

There are two main implications of the robustness of the linear model. First, no one has as yet proposed a better data organizing principle for organisms to have been endowed with throughout their evolutionary history; it would have provided robust inferences that no other procedure known to us even now can match. Second, if organisms evolved this robust organizing principle, they would not have to learn to assign correct weights among proximal and distal variables; approximately correct judgments would ensue in any event. Indeed, were the organism not already equipped with such a robust cognitive principle, it would be very difficult for any individual to learn in a stochastic environment. This is because the uncertain environment would not send back reliable information precise enough to support non-probabilistic inferences.

That conclusion is consistent with the well-established finding that our social perception is no more than mediocre in its accuracy, and, perhaps, why 50 years of research on clinical judgment shows that it is no more accurate than a linear model (Dawes, Faust, \& Meehl, 1989; Gardner, Lidz, Mulvey, \& Shaw, 1996; Hammond, 1955; Meehl, 1954). In short, any organism endowed with a robust domain-independent linear model as a cognitive system to be used for the purpose of inductive inference would be at a distinct advantage relative to any organism that relied exclusively upon domain-specific processes. Moreover, any organism so endowed would not have to learn to improve its judgments, nor is it likely, given the uncertainty in the environment that it could markedly improve them in any event. "Cognitive robustness" thus defines what is critically functional and is directly related to the ability to survive and reproduce in a probabilistic environment.

\subsection{Domain-dependence and the specificity of cues and means}

It should be clear from the above discussion of robustness that, within the Brunswikian framework, domain independence refers to the organizing principle (the linear model) used to process the data acquired from various multiple fallible indicators (Cooksey, 1996; Hammond, 1996, 2000). The content of the indicators is left unspecified because the process is largely, but not completely, general over the content of indicators. However, neither the ecological validities nor functional utilizations of the indicators are themselves domain-independent. This circumstance is recognized in the Brunswikian research of the 1960s and made evident by the wide variety of indicators used in numerous studies of multiple cue probability learning and "policy capturing" (Adelman, 1981; Brehmer \& Joyce, 1988; Cooksey, 1996; Holzworth, 2001).

In contrast, after specifying how to make use of the hypothetico-deductive approach to search for psychological mechanisms relevant to evolutionary psychology, Cosmides (1989) states: "Evidence for the existence of such mechanisms is: (1) reasoning performance is altered depending on what content the subject is asked

Author version. Final version published in Intelligence, v.34: 211-227 available at www.sciencedirect.com/science/article/pii/S016028960500022X.

DOI: $10.1016 / j$.intell.2005.03.006 
to reason about; and (2) such reasoning performance is altered by specific content in the predicted adaptive direction" (p. 190-191). Hirschfeld and Gelman (1994) also state: "A growing number of researchers now claim that many cognitive abilities are specialized to handle specific types of information" (see also Cosmides \& Tooby in Barkow, Cosmides \& Tooby, 1992; Cummins \& Allen, 1998).

These statements clarify one of the primary reasons that there has been so much disagreement. Brunswik focused on the formal nature of the cognitive process, while Cosmides examined the content of the cues or target object(s). As a result of these two restrictions of interest, very different theories arose. As Brunswik himself pointed out, however, specifying that the process by which indicators are organized is domain-independent does not exclude the idea that the indicators themselves can be domain-dependent. Of course, saying that an organism is "endowed" with both robust, domain-independent cognitive system and domaindependent mechanisms leaves open the question of how those endowments came about.

\section{A two-parameter model of preparedness and plasticity}

One of the most historically persistent questions in the study of cognitive development is whether and to what extent information is either genetically programmed or experientially acquired. Although the Nature/Nurture debate has presumably been resolved by a general consensus that both genetic and environmental sources of information contribute to and somehow interact in the process of behavioral development, exactly how these complementary contributions combine and how they can be expected to interact is something that has not been adequately specified.

Traditionally, the debate between ethologists, who have generally favored Nature over Nurture, and comparative psychologists, who have generally favored Nurture over Nature, has relied on typological conceptualizations of genetic versus environmental sources of information such as "instinct" and "learning". Contemporary theorists no longer consider "instinct" and "learning" to be mutually exclusive categories, but the currently popular view is that these phenomena lie instead along a single continuum. This one dimension has been variously labeled biological "canalization" (Waddington, 1957), "preparedness" (Seligman, 1970; Seligman \& Hager, 1972), "restrictedness" (Alcock, 1989), relative "closure" (Mayr, 1974) or "constraint" (Garcia \& Ervin, 1968; Garcia, Hankins, \& Rusiniak, 1974). These different names merely reflect differing emphases upon what can be regarded as complementary aspects of the same hypothetical process. Although this continuum theory represents a great advance over the strictly dichotomous prior view, it remains deficient, regardless of one's terminological preference. The correct location of many learning mechanisms along this theoretical continuum is problematical because different characteristics of the same phenomena often appear to straddle multiple distinct loci on the proposed dimension. For example, recent research has reported a number of unusual operating characteristics in the learning mecha-

Author version. Final version published in Intelligence, v.34: 211-227 available at www.sciencedirect.com/science/article/pii/S016028960500022X.

DOI: $10.1016 /$ j.intell.2005.03.006 
nisms of many invertebrates. Among these functional anomalies is extremely rapid learning in from a few to a single conditioning trial, as well as an apparent blurring of traditional distinctions between associative and nonassociative conditioning. Although some of these phenomena can be forced to fit within the framework of traditional learning paradigms, it has become reasonable to question whether there are indeed systematic qualitative differences between vertebrate and invertebrate learning mechanisms or whether a more inclusive theoretical framework can and should be developed. The hierarchical stochastic model for the optimal specification of prepared association strength proposed by Figueredo (1992, 1995, 2000) would indicate that not one but two independent parameters must be considered. These parameters, which we call preparedness and plasticity, model the ecological validity and temporal stability of the prepared association across evolutionary, ecological, and developmental times.

\subsection{Nested orders of magnitude in time}

To discuss these various validities and utilizations within the framework of modern evolutionary theory it is useful to break up the continuous stream of time into three hierarchically nested functional orders of magnitude: (1) evolutionary time, (2) ecological time, and (3) developmental time. Evolutionary time is the time scale required for normal evolutionary mechanisms, such as natural selection, to operate. Significant environmental change may occur over evolutionary time. Within evolutionary time, ecological time is the time frame, within which systematic environmental change may be considered virtually negligible. Over ecological time, therefore, there is assumed to be no significant evolutionary change. Within ecological time, developmental time is the time frame required for the normal lifetime development of the individual organism. Over developmental time, therefore, there is assumed to be no systematic ecological change or relocation across distinct ecologies for all but very long-lived species. Ecological time is therefore the cross-section of evolutionary time during which the environment can be considered fairly stable; a dynamic length of evolutionary time can thus be considered as a set (or Riemann Sum) of successive static frames of ecological time. Developmental time is the cross-section of ecological time in which the individual organism has to interact with and presumably adapt to that environment; a length of ecological time can thus usually be considered as a set of successive sections of developmental time. It is with respect to the relationships between these nested time scales that ecological validities and functional utilizations must be considered.

\subsection{Behavioral adaptation}

From the perspective of Probabilistic Functionalism, the essence of behavioral adaptation is the matching of the functional utilizations of the organism to the corresponding ecological validities of the environment. The critical question resides in precisely how that may be accomplished. From this perspective, the evolution of

Author version. Final version published in Intelligence, v.34: 211-227 available at www.sciencedirect.com/science/article/pii/S016028960500022X.

DOI: $10.1016 /$ j.intell.2005.03.006 
a biologically "prepared" association may be considered that of a genetically preprogrammed functional utilization. If the instantaneous ecological validity of the same association at any given point in time can be represented by a point estimate correlation coefficient, the mean value of that correlation over any given stretch of evolutionary time represents the average ecological validity of the association over a stochastic distribution representing many successive sections of ecological time. Because this average equals the expected value for any given section of ecological time, it specifies the point estimate for functional utilization or prepared association strength that would be selectively optimal.

Over evolutionary time, however, the variance in ecological validity about that mean correlation represents the temporal instability in the ecological validity of the association over the multiple successive sections of ecological time. Because this variance about the mean thus equals the degree of uncertainty about the overall expected value for any given section of ecological time, it implies some potential for local temporal mismatch between the instantaneous ecological validity and the evolved functional validity of the prepared association. Although the mean ecological validity over evolutionary time represents the global optimum point estimate for functional utilization in the long run, individual organisms need to survive in the short run. This creates selective pressure for behavioral plasticity.

Learning may be considered an evolved heuristic mechanism for the individual determination of critical ecological validities within developmental time. Such behavioral plasticity will be selected when the variance about the mean correlation over evolutionary time generates excessive chance of mismatch between the changing ecological validities and the evolved functional utilization of a prepared association, which might otherwise remain fixed at a constant value. The average discrepancy between the locally and the globally optimal behavior then imposes unacceptable opportunity costs on the individual within developmental time. Of course, learning itself takes time and there is an opportunity cost associated with iteratively approaching the locally optimal functional utilization at any finite rate, but this cost may be much less than that of being permanently fixed at a suboptimal value. It is the nature of this tradeoff which distinguishes this overtly statistical model from contemporary mainstream theories based on the unidimensional continuum between instinct and learning described above.

\subsection{Models of preparedness and plasticity}

Previous considerations of the costs of behavioral plasticity have inadvertently adopted the obsolete tabula rasa model of learning. They read as if abandoning fixed instinct required the complete sacrifice of biological preparedness. The implied representation of the tradeoffs involved misses the basic statistical distinction between the mean and the variance of a distribution. In a stochastic model, the quantitative implication of the tabula rasa assumption is that the learning process for an association must iteratively approach any final endpoint from a starting value of zero. This assumption is extremely consequential in its quantitative implications

Author version. Final version published in Intelligence, v.34: 211-227 available at www.sciencedirect.com/science/article/pii/S016028960500022X.

DOI: $10.1016 / j$.intell.2005.03.006 
because it greatly inflates the estimated cost of learning. Anyone who has used iterative estimation procedures on a computer can attest to the relative folly of starting at a "default" value of zero if any reasonable alternative values are available. The concept of biological "constraints" upon plasticity as a partial solution is a good one, but it restricts evolution to the negative role of setting limits to dispersion and ignores the positive role of providing more specific information on central tendency. Apparently forgotten is the fact that, whatever the magnitude of the variance, the best initial point estimate is still the population mean. This consideration implies that unless the mean ecological validity is indeed exactly zero over evolutionary time, learning processes in general should optimally proceed iteratively from a biologically prepared nonzero value. It is important to note here that zero is not an arbitrary value but represents a correlation coefficient of zero, indicating no significant ecological validity or functional utilization.

In this theory, the mean ecological validity coefficient over evolutionary time would thus specify the optimal initial estimate or starting potency of the prepared association; the variance of that correlation would specify the optimal margin for error or prepared plasticity of the association. These two parameters may vary independently to produce selective pressures not correctly modeled by a unidimensional continuum. Current theory on the relative costs and benefits of "closed" versus "open" developmental programs, however, confound these two dimensions. A high mean ecological validity is inappropriately linked to a low temporal instability (yielding a closed "instinct" solution); a high temporal instability is likewise linked to a low mean ecological validity (yielding an open "learning" solution). This may produce misleading conclusions about optimal tradeoffs in developmental strategy. Other than due to the boundedness of correlations at the upper and lower limits, there is no reason to suppose that these parameters must covary inversely over most of their range.

The sampling distribution of the correlation coefficient has a variance which is an inverse function of that coefficient. The expected variance of this theoretical distribution, however, may be applied to the distribution of validity coefficients in developmental times, nested within ecological times, but not to that of validity coefficients in ecological times, nested within evolutionary time. Because, by definition, the value of the population parameter for the correlation coefficient, called $\rho$, is held to be constant during any given section of ecological time, the idiosyncratic experience of any individual organism within its own developmental time may be modeled as a random sample from a population of such values, with a mean of $\rho$ and a variance equal to that of the theoretical sampling distribution. This model implies that an individual organism's experience, as a sample statistic, of a higher ecological validity, as the corresponding population parameter, is expected to be more consistent over iterative learning "trials" than that of a lower one, due entirely to the statistical characteristics of the sampling distribution of $\rho$. This purely stochastic effect would seem to facilitate more rapid learning of higher validity associations within developmental time, and might help account for some of the unusually rapid learning effects found in many species.

Author version. Final version published in Intelligence, v.34: 211-227 available at www.sciencedirect.com/science/article/pii/S016028960500022X.

DOI: 10.1016/j.intell.2005.03.006 
Nevertheless, the distribution of validity coefficients in ecological times, nested within evolutionary time, is not correctly modeled by the sampling distribution of $\rho$. This is because true ecological changes over time may represent systematic and persistent effects and not the purely random and serially independent processes normally assumed by sampling theory. Thus, although an added random variance component may also be present, the variance of ecological validities over evolutionary time may be otherwise completely unrelated to the sampling variance of the mean coefficient over evolutionary time, depending instead upon the average rate of true environmental change. Independent variance component models of this kind have been developed and successfully applied in both Meta-Analysis (e.g., Hedges \& Olkin, 1985) and Generalizability Theory (e.g., Shavelson, Webb, \& Roley, 1989).

This two-parameter model, therefore, mitigates the alleged conflict between biological preparedness and behavioral plasticity. It permits the possibility that an association can be both highly genetically prepared and highly developmentally plastic. Thus, as William James (1890) noted over a century ago, a highly sophisticated species might have more instincts as well as more learning abilities. Logically, it also permits the interesting situation where an ecological validity has a population mean close to zero and a negligible variance around it. This state of affairs would naturally select a prepared functional utilization virtually fixed at zero. Perhaps this represents the correct quantitative model for the great difficulty of conditioning certain associations documented in the taste-aversion literature (Garcia \& Ervin, 1968; Garcia et al., 1974). Fig. 2 displays all four logical possibilities predicted by this model. Cues or means with low mean ecological validity over evolutionary time are shown distributed about a central tendency of zero; those with high mean ecological validity are shown as displaced to the right. Cues or means with low variance in ecological validity over evolutionary time are distinguished by a lesser dispersion of their distribution; those with high variance are shown as wider curves. This four-way typology is for conceptual illustration only, and is not intended to imply any restrictions on the continuous distributions of the two freely varying parameters.

\subsection{Implications of the two-parameter model}

The traditional belief that substantial biological preparedness somehow precludes simultaneous behavioral plasticity leads to the mistaken notion that the environmentally induced modification of a biologically prepared response is something less than "true" learning and should be distinguished by special labels such as "alpha conditioning". Instead, we refer to it as "Jamesian learning" (see James, 1890), because it retains a complementary role for elements of "instinct", while remaining a form of true learning. According to the stochastic optimality model proposed here, it is the misguided search by the laboratory experimentalist for behaviors with no significant biological preparedness (indicating a mean ecological validity of zero in nature) which leads to a grossly unrepresentative sampling of

Author version. Final version published in Intelligence, v.34: 211-227 available at www.sciencedirect.com/science/article/pii/S016028960500022X.

DOI: $10.1016 / j$.intell.2005.03.006 
the population of likely natural environments and thus a systematically distorted view of the role of behavioral plasticity. Although Fig. 2 seems to imply that such circumstances are equally likely as not to occur, in natural ecologies to which the organism is adapted, it is highly unlikely that the mean ecological validities of any cues would be equal to zero. This is probably why the classical ethologists so emphasized the role of instinct when observing natural behavior in the field.

The application of a two-parameter model provides a better theoretical framework for understanding the extant empirical results and a less paradigmatically constrained heuristic for future research. This two-parameter model of preparedness and plasticity specifies a mechanism whereby domain-specific information content can be evolved within a purely domain-independent framework for cognitive development. This model therefore serves to reconcile the findings of contemporary evolutionary psychology with those of Brunswikian Probabilistic Functionalism. A single Brunswikian Evolutionary Developmental theory is created which may be used to account for the considerable bodies of evidence accumulated in favor of both the domain-dependent approach and the domain-independent approach to cognitive evolution and development.

\section{Unique predictions of the Brunswikian evolutionary de- velopmental theory}

When evaluating alternative scientific theories, it is important that one be able to derive unique and testable predictions. Examining learning within the evolutionary framework proposed here, a clear distinction emerges between the Brunswikian Evolutionary Developmental theory and the model of learning accepted by most contemporary theorists. Specifically, the pervasive dichotomy of associative and nonassociative learning is challenged by the evolutionary model. One need only look at the current learning literature to see the crux of the issue. For example, Vianna et al. (2000) write in their introductory paragraph:

"Long-term memories can be divided into associative and nonassociative memories depending on the mechanisms required for their formation. Associative memories are based on the acquisition of a predictive link between a specific event and a stimulus. Nonassociative memories are acquired when repeated or continuous exposure to a novel stimulus changes behavioral responses to it" (p. 333).

To clarify, associative behaviors are deemed such because two stimuli are paired: (1) an unconditioned stimulus (e.g., food) to which the animal responds (e.g., by salivating) without prior training, and (2) a conditioned stimulus (e.g., a bell) to which the animal has no prior response, must be presented contingently before the desired response is elicited (e.g., salivation at hearing the bell). Nonassociative learning is considered distinct from associative learning because only one stimulus is presented; an unconditioned stimulus (e.g., loud noise) to which the animal initially responds (e.g., by freezing) and then neglects to respond after several presentations.

Author version. Final version published in Intelligence, v.34: 211-227 available at www.sciencedirect.com/science/article/pii/S016028960500022X.

DOI: $10.1016 / j$.intell.2005.03.006 
Because contemporary learning theorists are only concerned with what is occurring within developmental time, the distinction between the two types of learning appears justified. Whereas in associative learning, the individual animal experiences a contingency that shapes the eventual response to the conditioned stimulus, the response observed to change in nonassociative learning is not seemingly related to a predictable outcome. However, from an evolutionary perspective, the reason that the animal responds to the unconditioned stimulus in the first place is precisely because it (or something so similar as to produce stimulus generalization) has been present, and moreover relevant, to the animal at some point in its evolutionary history. The animal's current behavior is thus the result of a functional utilization that has been prepared to a certain extent due to some degree of stability of the ecological validity of that cue or mean over evolutionary time. In short, there is a "predictive link between a specific event and a stimulus" as required by Vianna et al. (2000) for the formation of an associative memory. In the experimental setting the predictive or productive nature of that cue or mean then changes, and the organism adjusts its response (within the limit specified by the variance in the mean ecological validity) according to the new ecological validity present in the environment.

It should be noted that the previous argument is not meant to imply that there is no distinction between the associative and nonassociative cellular mechanisms that may underlie different cognitive processes. Rather, we suggest that the fact that we cannot observe the progression of biologically prepared associations should not prevent us from thinking of instinctive behaviors as governed by predictive links in the same way as those associations that we can observe forming within developmental time. In other words, from an evolutionary perspective all behavioral learning can be considered inherently associative in nature.

\subsection{Extinction and habituation}

From this discussion an empirical prediction clearly follows: behaviors previously regarded as representative of associative or nonassociative learning should show similarities. Two ideal behavioral mechanisms for comparison are extinction and habituation, commonly thought of as associative and nonassociative learning processes, respectively. Extinction is defined as a decrease in the expression of a learned response as a result of repeated presentations of the conditioned stimulus in the absence of the unconditioned stimulus (for reviews on extinction see Bouton, 2004; Delamater, 2004; Rescorla, 2001). To illustrate, Berman and Dudai (2001) described a procedure in which aversion to a taste was conditioned by pairing the stimulus with a chemical that induces nausea. Once this association was formed, the behavioral response was extinguished by presenting the same taste stimulus without the nausea-producing agent (Berman \& Dudai, 2001). Habituation is defined as a decrease in an innate response with repeated presentations of an unconditioned stimulus (for reviews on habituation see McSweeney \& Swindell, 2002; Rose \& Rankin, 2001; Thompson \& Spencer, 1966; Groves \& Thompson, 1970).

Author version. Final version published in Intelligence, v.34: 211-227 available at www.sciencedirect.com/science/article/pii/S016028960500022X.

DOI: $10.1016 / j$.intell.2005.03.006 
For example, Peterson and Squire (1977) reported that when mice heard the distress call of another mouse, it interrupted drinking behavior. However, this sound later failed to disrupt drinking after repetitive stimulation (Peterson \& Squire, 1977). Again, the distinction made by the contemporary learning theorist between extinction and habituation stems from what can be observed within developmental time. While in extinction two stimuli must be paired and a high asymptotic level of response reached before the response can be diminished through repeated presentations, habituation involves a response to only one stimulus that decreases from a high asymptotic level which is the baseline state of the behavioral response (see Hawkins \& Kandel, 1984, Fig. 4).

As discussed previously, however, recognizing only what occurs within developmental time can provide a skewed picture of the behavior being studied. Why is it that the animal responds to the unconditioned stimulus without prior training? Why is it that the animal does not respond to the conditioned stimulus until it is paired contingently with the unconditioned stimulus? Why are the responses to the conditioned and unconditioned stimuli observed to change after repeated presentations? To adequately answer these questions we must consider what has happened over ecological and evolutionary time, as well as developmental time. For example, as discussed above, Berman and Dudai (2001) describe a procedure in which they paired a taste with a nausea-producing agent to produce a conditioned aversion to the taste. The Brunswikian Evolutionary Developmental theory predicts that the animal does not respond to the taste, or, particularly, does not display aversion to it initially (i.e. the functional utilization is low) because the mean ecological validity of that cue as an indicator of sickness or danger has been relatively low over evolutionary time. In contrast, the cue of nausea has likely had high ecological validity over evolutionary time as an indicator of foods that should be avoided. When the taste and nausea are paired contingently in developmental time, the ecological validity of the taste changes such that it now reliably indicates that nausea will ensue after consumption of foods possessing that taste. The animal responds by changing its functional utilization such that the taste is subsequently avoided. When Berman and Dudai (2001) changed the ecological validity of the cue again by presenting the taste when it does not predict nausea, the animal readjusted its functional utilization such that it no longer avoided the taste (i.e., the response was extinguished).

Similarly, as mentioned above, Peterson and Squire (1977) presented a distress call to mice to disrupt drinking behavior and found that the mice were no longer disturbed after several presentations of the distress call. Brunswikian Evolutionary Developmental theory states that the animal responds to the distress call initially (i.e., its functional utilization is high) because the distress call has been a reliable cue in indicating distressed conspecifics (perhaps due to the presence of a predator in the vicinity of the calling mice). When the ecological validity of this cue is diminished by repeated presentation of the distress call in the absence of the initially expected negative consequence (such as the appearance of distressed conspecifics or a predator) the animal adjusts it functional utilization accordingly so that it is no

Author version. Final version published in Intelligence, v.34: 211-227 available at www.sciencedirect.com/science/article/pii/S016028960500022X.

DOI: $10.1016 /$ j.intell.2005.03.006 
longer interrupted when it hears the call (i.e., the response is habituated).

It is clear from the above discussion that there is a difference in the level of prepared functional utilizations of cues used in habituation and extinction procedures, and that this difference is based on the differing ecological validity of those cues over evolutionary time. However, the problem the brain must solve in extinction and habituation is essentially the same: to adjust behavior in accordance with changing environmental contingencies that are relevant to survival and reproduction. From this perspective, habituation can be thought of as extinction of a prepared association, or extinction thought of as habituation of a conditioned association. Either way, the task is accomplished in both instances by the domainindependent organizing principle of the Lens model, which functions across different contexts to allow the organism to extract information about, and respond to, changing domain-dependent environmental contingencies.

As to the empirical evidence supporting our prediction regarding habituation and extinction, we are not aware of any published studies in which the two behaviors have been directly compared experimentally. McSweeney and Swindell (2002) wrote an extensive review in which they compared the observable characteristics of habituation and extinction, such as effect of intertrial interval on the rate of each behavior and the phenomenon of spontaneous recovery, as demonstrated across a number of independent studies. The authors claimed that the results of their review showed remarkable overlap between the two behaviors, including a mathematical fit of extinction data to a model designed to explain the variance in data gathered on habituation. Citing behavioral overlaps years earlier, other researchers have gone so far as to claim that extinction and habituation might be mediated by the same underlying neural mechanisms (e.g., Hawkins \& Kandel, 1984; Thompson \& Spencer, 1966). Of course, it is still an open question whether these two behavioral processes, determined to be the same from a theoretical or behavioral perspective, are implemented by the brain using the same means. McKiernan and Figueredo (in preparation) review one avenue of the existing neurobiological literature on habituation and extinction in an attempt to begin answering this question. The evidence provided as to the presynaptic and postsynaptic signaling mechanisms involved in extinction and habituation shows that there are striking similarities between the two. In short, though further investigation is needed that directly compares these behavioral mechanisms, the preliminary evidence suggests that the prediction arising from the Brunswikian Evolutionary Developmental Theory as to the correspondence between habituation and extinction is supported.

\section{Conclusion}

In this paper, we have attempted to reconcile and synthesize the domain-dependent with the domain-independent approaches to cognitive evolution and development. We have presented a two-parameter model of preparedness and plasticity which specifies a mechanism whereby domain-dependent information content can be evolved

Author version. Final version published in Intelligence, v.34: 211-227 available at www.sciencedirect.com/science/article/pii/S016028960500022X.

DOI: $10.1016 /$ j.intell.2005.03.006 
within a purely domain-independent organizing framework for the processes of cognitive development. Within the framework of Brunswikian Probabilistic Functionalism, domain independence resides in the organizing principle used for processing the data acquired from various multiple fallible indicators, whereas domain dependence resides within the unique ecological validities and functional utilizations of the indicators themselves. This Brunswikian Evolutionary Developmental theory accounts for the considerable bodies of evidence accumulated in favor of both the domain-dependent approach and the domain-independent approaches. Our theory also provides a new way of thinking about old problems, such as the distinction between associative and nonassociative learning, which leads to the generation of unique and testable hypotheses. A prediction made by the Brunswikian Evolutionary Developmental theory, examined in detail elsewhere, is the hypothesis that habituation and extinction may represent the same behavioral process. The preliminary evidence available to date appears to support this assertion. Thus, we believe that this new theoretical perspective represents a productive direction in which to conduct further research in the hopes of making progress in the study of cognitive evolution and development.

\section{Acknowledgments}

The authors would like to thank Dr. Melinda Davis, Dr. Norman Davis, Marco HerreraValdez, Dr. James E. King, and Dr. W. Jake Jacobs for valuable discussions and comments regarding this manuscript and its content. Thanks also to Dr. Linda Gottfredson for her detailed review and her many helpful suggestions for revision.

Author version. Final version published in Intelligence, v.34: 211-227 available at wWw.sciencedirect.com/science/article/pii/S016028960500022X. 


\section{Figures}

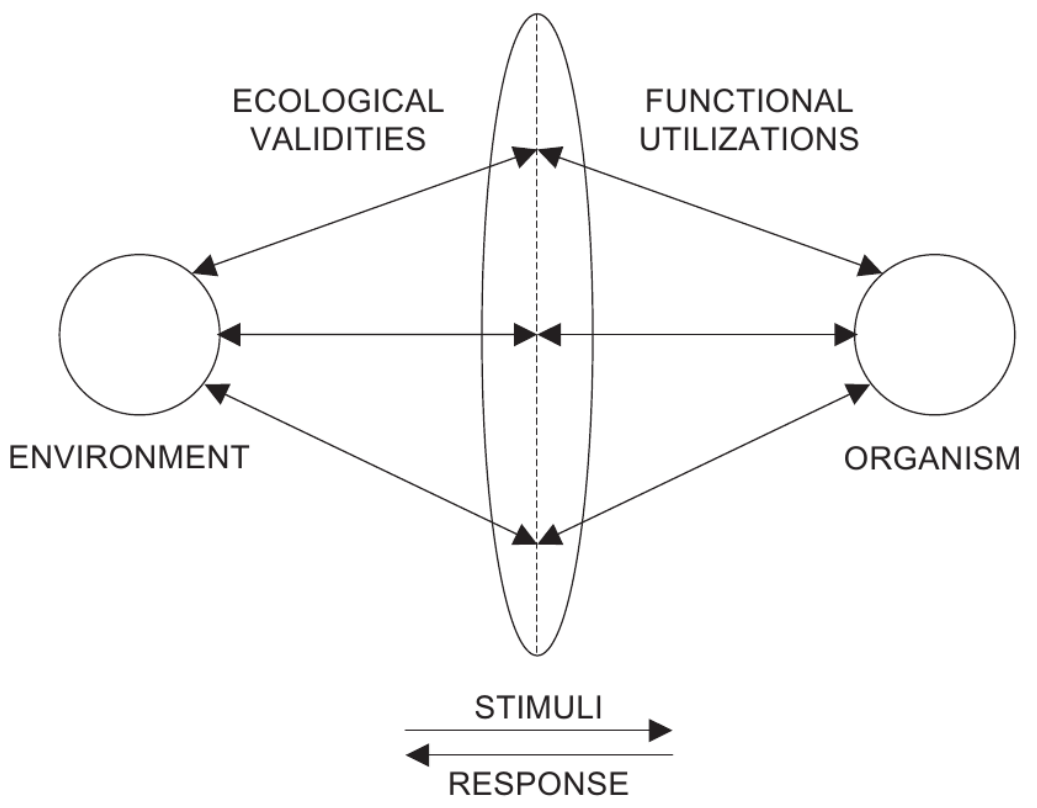

Figure 1: The Lens Model of Egon Brunswik.

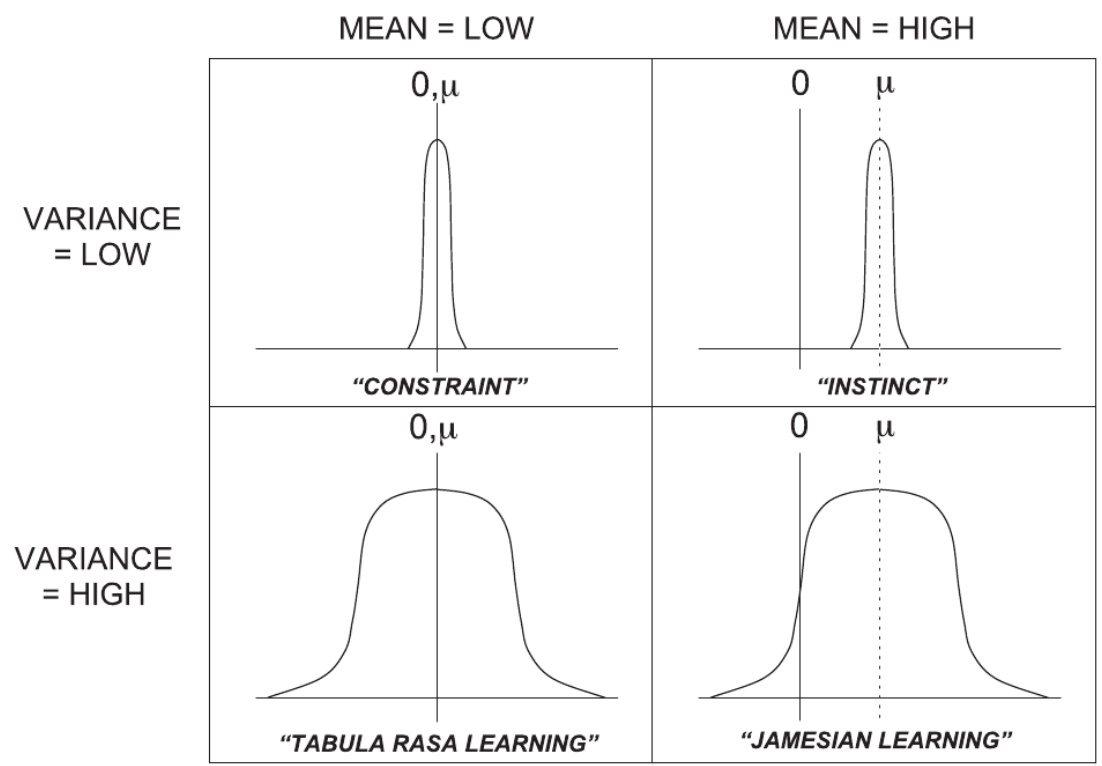

Figure 2: The two-parameter model of preparedness and plasticity. Note: There was an error in the published figure. Symbol 0 should be $\rho$, to correspond to text.

Author version. Final version published in Intelligence, v.34: 211-227 available at www.sciencedirect.com/science/article/pii/S016028960500022X.

DOI: 10.1016/j.intell.2005.03.006 


\section{References}

Adelman, L. (1981). The influence of formal, substantive, and contextual task properties on the relative effectiveness of different forms of feedback in multiple-cue probability learning tasks. Organizational Behavior and Human Performance 27(3), 423-442.

Albright, L. and T. Malloy (2001a). Brunswik's theoretical and methodological contributions to research in interpersonal perception. In K. Hammond and S. TR (Eds.), The essential Brunswik: Beginnings, explications, applications, pp. 328-332. New York: Oxford University Press.

Albright, L. and T. Malloy (2001b). Probability learning or partial reinforcement. In K. Hammond and S. TR (Eds.), The essential Brunswik: Beginnings, explications, applications, pp. 167-170. New York: Oxford University Press.

Alcock, J. and P. Farley (2001). Animal behavior: an evolutionary approach. Sinauer Associates Massachusetts.

Barkow, J., L. Cosmides, and J. Tooby (1995). The adapted mind: Evolutionary psychology and the generation of culture. Oxford University Press, USA.

Berman, D. and Y. Dudai (2001). Memory extinction, learning anew, and learning the new: dissociations in the molecular machinery of learning in cortex. Science 291(5512), 2417-2419.

Bouton, M. (2004). Context and behavioral processes in extinction. Learning \& Memory 11(5), 485-494.

Brehmer, B. (1988). Human judgment: The social judgment theory view, Volume 54. North Holland.

Brunswik, E. (1938). Das induktionsprinzip in der wahrnehmung. In H. Pieron and J. Meyerson (Eds.), l lieme Congres International de Psychologie a Paris 1937. Rapports et Comptes Rendus, pp. 346-347. Alcan.

Brunswik, E. (1952). The conceptual framework of psychology. Univ of Chicago Press.

Brunswik, E. (1955a). In defense of probabilistic functionalism: a reply. Psychological Review 62(3), 236-242.

Brunswik, E. (1955b). Representative design and probabilistic theory in a functional psychology. Psychological Review 62(3), 193.

Brunswik, E. (1956). Perception and the representative design of psychological experiments ( $2 d e d$.). University of California Press.

Brunswik, E. (1957). Scope and aspects of the cognitive problem. In H. Gruber, K. Hammond, and R. Jessor (Eds.), Contemporary approaches to cognition: a symposium held at the University of Colorado, pp. 5-31. Harvard University Press.

Brunswik, E. and H. Herma (1951). Probability learning of perceptual cues in the establishment of a weight illusion. Journal of Experimental Psychology 41(4), 281.

Author version. Final version published in Intelligence, v.34: 211-227 available at www.sciencedirect.com/science/article/pii/S016028960500022X.

DOI: $10.1016 /$ j.intell.2005.03.006 
Buss, D. et al. (1989). Sex differences in human mate preferences: Evolutionary hypotheses tested in 37 cultures. Behavioral and brain sciences 12(1), 1-49.

Byrne, R. and R. Byrne (1995). The thinking ape: Evolutionary origins of intelligence, Volume 129. Oxford University Press Oxford.

Cohen, J. (2003). Applied multiple regression/correlation analysis for the behavioral sciences, Volume 1. Lawrence Erlbaum.

Cooksey, R. (1996). Judgment analysis: Theory, methods, and applications. Academic Press.

Cosmides, L. (1989). The logic of social exchange: Has natural selection shaped how humans reason? studies with the wason selection task. Cognition 31(3), 187-276.

Cummins, D. and C. Allen (1998). The evolution of mind. Oxford University Press, USA.

Dawes, R. and B. Corrigan (1974). Linear models in decision making. Psychological Bulletin 81(2), 95.

Dawes, R., D. Faust, and P. Meehl (1989). Clinical versus actuarial judgment. Science 243(4899), 1668.

Delamater, A. (2004). Experimental extinction in pavlovian conditioning: Behavioural and neuroscience perspectives. Quarterly Journal of Experimental Psychology Section B 57(2), 97-132.

Doherty, M. (2001a). Demonstrations for Gestalt psychologists: Psychology without a subject. In K. Hammond and S. TR (Eds.), The essential Brunswik: Beginnings, explications, applications, pp. 211-213. New York: Oxford University Press.

Doherty, M. (2001b). The realistic accuracy model and Brunswik's approach to social judgment. In K. Hammond and S. TR (Eds.), The essential Brunswik: Beginnings, explications, applications, pp. 365-369. New York: Oxford University Press.

Figueredo, A. (1992). Preparedness and plasticity: A stochastic optimality theory. In Human Behavior and Evolution Society: Fourth Annual Meeting. Albuquerque, NM.

Figueredo, A. (1995). A stochastic optimality theory of preparedness and plasticity. Behavioral and Brain Sciences 18(02), 300-301.

Figueredo, A. (2000). A Brunswikian optimality model for the evolutionary psychology of preparedness and plas- ticity. In Brunswik 2000, Max Planck Institut fur Bildungsforschung, Berlin, Germanyq.

Gardner, W., C. Lidz, E. Mulvey, and E. Shaw (1996). Clinical versus actuarial predictions of violence in patients with mental illnesses. Journal of Consulting and Clinical Psychology 64(3), 602.

Gigerenzer, G. (1991). From tools to theories: A heuristic of discovery in cognitive psychology. Psychological review 98(2), 254.

Gillis, J. and F. Bernieri (2001). The perception and judgment of rapport. In K. Hammond and S. TR (Eds.), The essential Brunswik: beginnings, explications, applications, pp. 380-83. New York: Oxford University Press.

Author version. Final version published in Intelligence, v.34: 211-227 available at www.sciencedirect.com/science/article/pii/S016028960500022X.

DOI: 10.1016/j.intell.2005.03.006 
Groves, P. and R. Thompson (1970). Habituation: A dual-process theory. Psychological Review; Psychological Review 77(5), 419.

Hammond, K. (1955). Probabilistic functioning and the clinical method. Psychological Review; Psychological Review 62(4), 255.

Hammond, K. (1996). Human judgment and social policy: Irreducible uncertainty, inevitable error, unavoidable injustice. Oxford University Press, USA.

Hammond, K. (2000). Judgments under stress. Oxford University Press, USA.

Hawkins, R. and E. Kandel (1984). Is there a cell-biological alphabet for simple forms of learning? Psychological review 91(3), 375.

Hedges, L. and I. Olkin (1985). Statistical methods for meta-analysis.

Hirschfeld, L. and S. Gelman (1994). Mapping the mind: Domain specificity in cognition and culture. Cambridge Univ Pr.

Holzworth, R. (2001). Multiple cue probability learning. In K. Hammond and S. TR (Eds.), The essential Brunswik: beginnings, explications, applications, pp. 348-350. New York: Oxford University Press.

James, W. (1890). The principles of psychology. Henry Holt and Company.

Mayr, E. (1974). Behavior programs and evolutionary strategies: Natural selection sometimes favors a genetically" closed" behavior program, sometimes an" open" one. American Scientist 62(6), 650-659.

McKiernan, E. and A. Figueredo. Common neural signaling mechanisms underlie habituation and extinction. (in preparation).

McSweeney, F. and S. Swindell (2002). Common processes may contribute to extinction and habituation. Journal of general psychology 129(4), 364-400.

Meehl, P. (1954). Clinical versus statistical prediction: A theoretical analysis and a review of the evidence. University of Minnesota Press.

Mumpower, J. (2001). Brunswikian research on social perception, interpersonal learning and conflict, and negotiation. In K. Hammond and S. TR (Eds.), The essential Brunswik: beginnings, explications, applications, pp. 388-393. New York: Oxford University Press.

Peterson, G. and L. Squire (1977). Cerebral protein synthesis and long-term habituation. Behavioral biology 21(3), 443-449.

Petrinovich, L. (1979). Probabilistic functionalism: A conception of research method. American Psychologist 34(5), 373.

Rescorla, R. (2001). Experimental extinction. In R. Mowrer and S. Klein (Eds.), Handbook of contemporary learning theories, pp. 119-154. Lawrence Erlbaum Assoc.

Rose, J. and C. Rankin (2001). Analyses of habituation in caenorhabditis elegans. Learning \& Memory 8(2), 63-69.

Author version. Final version published in Intelligence, v.34: 211-227 available at wWw.sciencedirect.com/science/article/pii/S016028960500022X.

DOI: 10.1016/j.intell.2005.03.006 
Seligman, M. (1970). On the generality of the laws of learning. Psychological review 77(5), 406.

Seligman, M. and J. Hager (1972). Introduction. In M. Seligman and J. Hager (Eds.), Biological boundaries of learning. Appleton-Century-Crofts.

Shavelson, R., N. Webb, and G. Rowley (1989). Generalizability theory. American Psychologist 44(6), 922.

Thompson, R. and W. Spencer (1966). Habituation: a model phenomenon for the study of neuronal substrates of behavior. Psychological review 73(1), 16.

Tolman, E. and E. Brunswik (1935). The organism and the causal texture of the environment. Psychological Review 42(1), 43.

Vianna, M., M. Alonso, H. Viola, J. Quevedo, F. De Paris, M. Furman, M. de Stein, J. Medina, and I. Izquierdo (2000). Role of hippocampal signaling pathways in long-term memory formation of a nonassociative learning task in the rat. Learning \& Memory 7(5), 333-340.

von Schantz, T., H. Wittzell, G. Göransson, and M. Grahn (1997). Mate choice, male condition-dependent ornamentation and mhe in the pheasant. Hereditas 127(1-2), 133140 .

Waddington, C. and H. Kacser (1957). The strategy of the genes. Allen and Unwin.

Wilton, D. (1968). Oviposition site selection by the tree-hole mosquito, aedes triseriatus (say). Journal of Medical Entomology 5(2), 189-194.

Author version. Final version published in Intelligence, v.34: 211-227 available at www.sciencedirect.com/science/article/pii/S016028960500022X.

DOI: $10.1016 /$ j.intell.2005.03.006 